\title{
Theoretical Analysis and Performance Comparison of Multi- carrier Waveforms for 5G Wireless Applications
}

\author{
Jamal Mestoui ${ }^{1}$, Mohammed El Ghzaoui ${ }^{1,2}$ \\ ${ }^{1}$ ISNET Team, IMAGE Laboratory, EST \\ Moulay Ismail University of Meknes, Meknes, 11201, MOROCCO \\ ${ }^{2}$ FSDM \\ Sidi Mohamed Ben Abdellah University, Fes, MOROCCO \\ Jamal Mestoui, \\ mestoui@hotmail.com
}

DOI: https://doi.org/10.30880/ijie.2021.13.06.019

Received 27 March 2020; Accepted 01 May 2021; Available online 31 August 2021

\begin{abstract}
Fifth generation (5G) wireless technology is a new wireless communication system that must meet different complementary needs: the high data rate for mobile services, low energy consumption and long-range for connected objects, low latency to ensure real-time communication for critical applications and, high spectral efficiency to improve the overall system capacity. The waveforms and associated signal processing present a real challenge in the implementation of each generation of wireless communication networks. Different research works have discussed this topic; but, these works are limited to the Filter-Bank Multi-Carrier (FBMC), Universal Filtered Multi-Carrier (UFMC), and Filtered Orthogonal Frequency-Division Multiplexing (F-OFDM) waveforms. In this work, we review the technical basics of the physical layer for the LTE system which uses, OFDM modulation for the downlink and the single carrier frequency division multiple access (SC-FDMA) technique for the uplink. Besides, this paper presents the diverse waveforms candidate for $5 \mathrm{G}$ systems, including, FBMC, UFMC, and FOFDM, and compares these techniques with Constant Envelope-OFDM (CE-OFDM), which is an advantageous form regarding power consumption, especially for battery-powered devices. Simulations are carried out to compare the performance of the OFDM, CE-OFDM, F-OFDM, UFMC, and FBMC in terms of power spectral density (PSD) and Bit Error Rate (BER). It has been demonstrated that (CE-OFDM) constitutes a more efficient solution in terms of energy consumption than the other technics. By comparison with the other technics, the CE-OFDM scheme performances, in terms of BER and PSD, are controllable by the values of its parameters (M, h, and J). This advantage gives designers of the system to consider the mutual choice between spectral efficiency and the BER. Moreover, the (F-OFDM), (UFMC), and (FBMC) schemes could constitute a more efficient solution in terms of power spectral density. It can be concluded from this paper that CE-OFDM wave form gives the best performance in terms of power consumption by reducing the Peak to Average Power Ratio (PAPR) associated with the OFDM system and FBMC is an efficiency technique to reduce the inter-carrier interference (ICI).
\end{abstract}

Keywords: 5G, OFDM, OOB, CE-OFDM, FBMC, UFMC, F-OFDM

\section{Introduction}

Over the past decade, the need for broadband wireless communications services has increased. These services are confronted with numerous energy and spectral constraints on the implementation of networks and terminals. 
Indeed, $4 \mathrm{G}$ is currently the latest generation of cellular technologies, the fifth generation (5G) will be used for the future communication system. Like 3G technologies, Long Term Evolution (LTE) and LTE-Advanced have used a wide range of mechanisms to support the growing load of mobile data users. But unlike $3 \mathrm{G}$, using Code division multiple access (CDMA) multiplexing, Orthogonal Frequency-Division Multiple Access (OFDMA) used in 4G can drastically reduce the interference experienced by transmissions, greatly improving system performance. $5 \mathrm{G}$ brings us from the design of networks connecting mobile devices only to systems that connect different types of devices operating at high speed. The main features of $5 \mathrm{G}$ are high throughput, improved spectrum efficiency, reduced latency, better mobility support, and high connection density. To support the increased $5 \mathrm{G}$ bandwidth requirements, a new spectrum has been assigned to the $5 \mathrm{G}$ millimeter wave bands. $5 \mathrm{G}$ technology is designed to support data rates of several Gbps [1], [2]. To achieve this goal, one of the most difficult tasks of wireless transceivers is to generate millimeter waveforms (mmW) in the measurement band greater than $24 \mathrm{GHz}$ [3], [4] with reduced Peak to Average Power Ratio (PAPR), better energy efficiency and good spectral efficiency.

The switch to $5 \mathrm{G}$ would be a significant break from $4 \mathrm{G}$ systems, such as LTE and Wi-Fi based on Orthogonal Frequency-Division Multiplexing (OFDM), as a multi-carrier modulation technique. This technique has important advantages, its flexible resource allocation and simplified equalization. Even these important advantages, OFDM has disadvantages. OFDM is based on the use of rectangular pulses in the time domain, which leads to a slowly decaying behavior in the frequency domain; this makes OFDM unsuited for use in fragmented spectrum scenarios, where strict constraints on the out-of-band (OOB) levels are to be fulfilled. On the other hand, in 4th generation systems, control of out-of-band emissions is performed by filtering the whole OFDM signal. Both solutions unfortunately lead to a loss in spectral efficiency, since in the former case some of the available subcarriers are actually not modulated, while in the latter case we need a longer cyclic prefix to combat the time dispersion induced by the filtering operation. Moreover, the need for a long cyclic prefix (CP) in heavy multipath environments is then another factor that degrades the system spectral efficiency. Likewise, the need for strict frequency and time synchronization among blocks and subcarriers in order to maintain orthogonality is a requirement that does not match well with the IoT scenario, wherein many devices have to access the channel with short data frames. Furthermore, Synchronization is also a key issue in the uplink of a cellular network wherein different mobile terminals transmit separately [5], and in the downlink, when base station coordination is used [6]. Additionally, OFDM signals may exhibit large PAPR values [7], and this has a clear impact on the system energy efficiency.

In order to meet the different requirements of $5 \mathrm{G}$ in terms of energy efficiency, spectral efficiency and to meet the needs of users of different services offered by $5 \mathrm{G}$, many waveforms have been proposed with particular design parameters.

For high spectral efficiency, many filter-based waveforms have been widely studied recently. The advantage of filter-based waveforms is that they use different designs to support asynchronous transmission by reducing OOBs [8]. In general, the filter-based waveforms can be divided into three types: subcarrier filtering sub-band filtering and fullband filtering. The Subcarrier Filtering approach is used in Filter-Bank Multi-Carrier (FBMC) [9], [10]. In this system, any modulation type might be employed. However, it can suffer from performance degradation in harsh environment such as large Doppler spread or channel delay spread [9]. In addition, the filter banks used in FBMC are taken from a prototype filter that controls the system performance, such as inter-symbol interference (ISI) and inter-carrier interference (ICI) [10]. The sub-band filtering is applied to Universal Filtered Multi-Carrier (UFMC) [11], [12]. This later can offer many advantages like a spectral efficiency similar to FBMC with less complexity, and lower power back-Off than for an OFDM system. The full-band filtering exploited by Filtered-OFDM (F-OFDM) [13], [14] is a technique used to reduce the input back off power (IBO) associated with OFDM modulation.

For good energy efficiency, the amplification of the OFDM signal with constant envelope is effective. This is, done at a minimum IBO and does not cause spectral widening [15]. By phase modulation, Constant Envelope-OFDM incorporates the information contained in a high PAPR message at the carrier phase rather than at its amplitude, giving a constant envelope RF signal -an optimal 0 dB PAPR-. The addition of a spectral precoder in the CE-OFDM block allows the reduction of powers of the side lobes [16]. The solutions proposed in [17] allow the reduction of the complexity of CE-OFDM system. However, to improve the spectral efficiency of CE-OFDM, a Quasi-CE-OFDM scheme is proposed in [18] which use a phase/ quadrature modulator. The constant envelope waveform can be applied for digital transmission via PLC channels [19]. More applications of CE-OFDM are detailed in [20]. In [21], [22], the authors proposed a CE-OFDM-CPM scheme using Multiple-Input Multiple-Output (MIMO) to increase the capacity of the OFDM system.

This article aims to use those solutions to reduce energy consumption and increase the use of bandwidth in the 5G network. Our objective is, on one hand, to increase the spectral efficiency, on the other hand to increase the energy efficiency of the systems, which constitutes a fundamental challenge, these two objectives often being contradictory. Simulations are conducted to complete this study. They allow comparison of the proposed systems in terms of Power Spectral Density (PSD) and in terms of bit error rate (BER). To illustrate these results, the BERs versus signal-to-noise ratio (SNR) are given for the proposed systems.

This paper is organized as follows: section 2 presents the related works. The transition techniques used in the $4 \mathrm{G}$ LTE standard are given in section 3. In the section 4, the basic principles and characteristics of various candidate 
waveforms for 5G technology are presented. In section 5, the simulation parameters and results are presented and discussed. Finally, we closed the paper with brief conclusions in section 6.

\section{Related Works}

The demand for throughput increases significantly each year with the emergence of new applications and services. These new trends push $4 \mathrm{G}$ to its limits. Thus, the 5th generation of mobile networks $(5 \mathrm{G})$, currently being standardized, will have to provide effective solutions to these new challenges. This new generation plans to introduce new types of services for these emerging applications, while improving the conventional mobile communications service in terms of throughput, latency and network coverage. In this context, several techniques are being explored to meet the requirements of 5G. Among the most promising techniques in the physical layer, new multi-carrier waveforms are proposed. Many researches warried out on multi-carrier waveforms in order to propose a souhaitble one for 5G application.

Indeed, the majority of the work related to this subject is based on the comparison of the waveforms FBMC, UFMC, F-OFDM and Generalized Frequency Division Multiplexing (GFDM) with the popular waveform OFDM. In [23], authors present a comprehensive comparison between these waveforms in terms of spectral efficiency, complexity and compatibility with MIMO technique. The work presented in [24] is limited to the study of the UFMC scheme, its performance is evaluated in terms of spectral efficiency, BER, PAPR, Carrier Frequency Offsite (CFO), time delay (TD) effects, etc. It has been demonstrated that the UFMC suffers from the same problems as other multi-carrier modulations, including a higher PAPR, affected by CFO, but it has its own advantages inherent such as delay insensitivity or energy efficiency, and the main negative factor of UFMC is ICI. In [25], authors tried to build a common framework based on the OFDM principle and derive these new waveforms. This derivation provides a new perspective that facilitates the direct understanding of channel equalization and the application of these new waveforms to multi-input multi-output channels. It also facilitates the derivation of new structures for a more efficient synthesis/analysis of these waveforms than those reported in the literature. In [26], several key requirements are presented to determine the merit of candidate waveform formats to meet $5 \mathrm{G}$ requirements in the mmWave ARoF architecture. An overview of the different waveforms suitable for this architecture is provided, discussing their advantages and disadvantages. In addition, a complete comparison in terms of different requirements is also presented in this document. Authors of [27] provided comprehensive analysis and comparison of typical waveforms (FBMC, UFMC, GFDM, F-OFDM). In addition to the basic principles of these waveforms, the authors also reveal the underlying characteristics. In [28], OFDM's downlink were strained and he demonstrated that Filter bank multicarrier (FBMC) could be a more impressive answer. The comparative study of the FBMC and OFDM was carried out in view of the power spectral densities reproduced using MATLAB. Based on this work author have concluded that the FBMC system eliminates the disadvantages of OFDM and can the candidate waveform for future systems 5G systems. In [29], authors review the concept of channel estimation in FBMC, OFDM-Offset Quadrature Amplitude Modulation (OFDMOQAM), UFMC and GFDM-OQAM waveforms as well as in other candidate waveforms for study in emerging $5 \mathrm{G}$ networks. In [30], authors compare the performance of candidate waveforms such as GFDM, BFDM, FBMC, UFMC, NOMA and with OFDM. Based on the analysis of the results and discussions, a new waveform is proposed that overcomes the disadvantages of previous waveforms in use. The proposed waveform was windowed and filtered OFDM. According to goyal et al. WFOFDM supports M-ary quadrature amplitude modulation (M-QAM) modulation and is a good waveform competitor which results in improved performance, reduced OOB emission in shorter window length (6 or 8) and increased spectral efficiency. Researchers have tried to make a comparison of different mutli-carrier waveform for $5 \mathrm{G}$ wireless applications in order to propose a completive one. In this direction, we have proposed a new waveform for $5 \mathrm{G}$ band which is CE-OFDM. This technique has the advantage to eliminate the PAPR associated with other waveforms as well it is a less power consumption which allows this technique to be desirable for battery-powered wireless systems.

\section{OFDM and SC-FDMA in 4G networks}

The current 4G standard, the LTE system, is based on the use of the OFDM modulation for the downlink and of the single carrier frequency division multiple access (SC-FDMA) technique for the uplink [31]. OFDM is an orthogonal block transmission scheme which, in ideal conditions, is not affected by inter-carrier interference and inter-symbol interference. Among the chief advantages of OFDM and OFDMA are: a)- The simplicity of implementation of the transceiver thanks to the fast Fourier transform (FFT) and inverse fast Fourier transform (IFFT) blocks, b)- The ability to counteract multi-path distortion, c)- The orthogonality of subcarriers, which eliminates inter-cell interference, d)Their easy coupling with adaptive modulation techniques And e)- The easy integration in multi-antennas hardware.

\subsection{Orthogonal Frequency Division Multiplexing (OFDM)}

OFDM is a modulation technique which has revolutionized the wireless communications industry. OFDM is a multicarrier scheme, which divides a wideband signal into multiple narrower bands of spectrum such that the aggregate 
signal does not suffer the total impact of the fading in a wideband multipath channel. This lends OFDM its inherent ability to enable high-speed communication on harsh wideband multipath channels without the need for complex channel equalization. This virtue has made OFDM a favorite modulation technique for $802.11 \mathrm{Wi}$ Fi protocols, and the 4G cellular standards.

OFDM makes to transmit a block of $N$ symbols during a period $T_{B}$ in parallel manner on $N$ subcarriers. Transmitting a cyclic prefix during a guard interval between successive blocks avoids the ISI. The interval duration, $T_{g}$, is designed such that $T_{g} \geq \tau_{\max }$ (channel's maximum propagation delay) so that the channel is absorbed in the guard interval and the OFDM block is uncorrupted.

The OFDM signal can be expressed as

$$
s(t)=\sum_{i}\left[\sum_{k=0}^{N-1} I_{i, k} e^{j 2 \pi f_{k} t}\right] g\left(t-i T_{B}\right)
$$

The pulse shape, $g(t)$, is typically rectangular:

$$
g(t)= \begin{cases}1, & 0 \leq t \leq T_{B} \\ 0, & \text { Otherwise }\end{cases}
$$

Notice that the $N$ data symbols $\left\{I_{i, k}\right\}_{k=0}^{N-1}$ are transmitted during the ith block. The set of complex sinusoids $\left\{e^{j 2 \pi f_{k} t}\right\}_{k=0}^{N-1}$ are referred to as subcarriers. The center frequency of the kth subcarrier is $f_{k}=k / T_{B}$ and the subcarrier spacing, $1 / T_{B} \mathrm{~Hz}$, makes the subcarriers orthogonal over the block interval, expressed mathematically as

$$
\begin{aligned}
\frac{1}{T_{B}} \int_{0}^{T_{B}}\left(e^{j 2 \pi f_{k 1} t}\right)^{*}\left(e^{j 2 \pi f_{k 2} t}\right) d t & =\frac{1}{T_{B}} \int_{0}^{T_{B}}\left(e^{j 2 \pi\left(f_{k 2}-f_{k 1}\right) t}\right) d t \\
= & \begin{array}{ll}
1, & k_{1}=k_{2} \\
0, & k_{1} \neq k_{2}
\end{array}
\end{aligned}
$$

where $(.)^{*}$ represents the complex conjugate operation, the subcarrier orthogonality can also be viewed in the frequency domain. Consider the Oth OFDM block:

$$
s(t)=\sum_{k=0}^{N-1} I_{0, k} e^{j 2 \pi f_{k} t}, \quad 0 \leq t \leq T_{B}
$$

During the OFDM block interval, the waveform is

$$
s(t)=\sum_{k=0}^{N-1} I_{k} e^{j 2 \pi f_{k} t}, \quad 0 \leq t \leq T_{B}
$$

where $\left\{I_{i, k}\right\}_{k=0}^{N-1}$ are the data symbols, $\left\{e^{j 2 \pi f_{k} t}\right\}_{k=0}^{N-1}$ are the subcarriers, $N$ is the total number of subcarriers, $f_{k}=k / T_{B}$ is the center frequency of the kth subcarrier and $T_{B}$ is the block period. The guard interval is defined during $-T_{g} \leq t<0$, where $T_{g}$ is the guard period. To transmit a cyclic prefix, the last $T_{g} \mathrm{~s}$ of the block is transmitted during the guard interval:

$$
s(t)=\sum_{k=0}^{N-1} I_{k} e^{j 2 \pi f_{k}\left(t+T_{B}\right)}=\sum_{k=0}^{N-1} I_{k} e^{j 2 \pi f_{k} t} e^{j 2 \pi k}=\sum_{k=0}^{N-1} I_{k} e^{j 2 \pi f_{k} t}
$$

where $-T_{g} \leq t<0$.

Notice that the above simplification is made due to the periodicity of the signal. Thus the OFDM signal having a guard interval with cyclic prefix is simply

$$
s(t)=\sum_{k=0}^{N-1} I_{k} e^{j 2 \pi f_{k} t}, \quad-T_{g} \leq t \leq T_{B}
$$

The received signal is

$$
\begin{aligned}
r(t) & =s(t) * h(\tau)+n(t) \\
& =\int_{-\infty}^{+\infty} h(\tau) s(t-\tau) d \tau+n(t) \\
& =\int_{0}^{\tau_{\max }} h(\tau) s(t-\tau) d \tau+n(t)
\end{aligned}
$$

where $h(\tau)$ is the time-invariant channel impulse response 1 and $n(t)$ is additive noise. 


\subsection{Single Carrier Frequency Division Multiple Access (SC-FDMA)}

Single Carrier Frequency Division Multiple Access (SC-FDMA) is a slightly different version of OFDM used in the uplink of LTE. It is a promising transmission technology for portable devices due to its low PAPR and a less sensitivity to frequency offset [32], [33]. The conventional SC-FDMA (C-SCFDMA) presented in Fig. 1(a) consists of $N_{a}$-Discrete Fourier Transform (DFT) and a subcarrier mapping followed by $N$-IFFT, where $N_{a}$ is the number of subcarriers allocated to a user and $N$ is the full IFFT size. Let $(m), m=0,1, \cdots, N_{a}$ - 1 , be modulated samples. The output of $N_{a}$-DFT is written as

$$
X_{d}(n)=\sum_{m=0}^{N_{a}-1} x(m) \exp \left[-j 2 \pi \frac{n m}{N_{a}}\right]
$$

The subcarrier mapping expands $(n)$ by zero padding as

$$
X_{c}(|n| N)=\left\{\begin{array}{lc}
X_{d}\left(\left|n-n^{*}\right| N_{a}\right), & n^{*}-\frac{N_{a}}{2} \leq n \leq n^{*}+\frac{N_{a}}{2}-1 \\
0 & \text { Otherwise }
\end{array}\right.
$$

where $n^{*}$ is the subcarrier shift corresponding to the distance between the direct current (DC) subcarrier and the center subcarrier in the allocation and $|k|_{N}=k \bmod N$ is modulo operation. The output of $N$-IFFT is written as

$$
y_{d}(k)=\frac{1}{N} \sum_{n=0}^{N-1} X_{c}(n) \exp \left[j 2 \pi \frac{n m}{N_{a}}\right], \quad 0 \leq k \leq N-1
$$

As system bandwidth grows, SC-FDMA transmitters face some challenges in implementation to meet the budget requirement of the processing time, where inverse fast Fourier transform (IFFT) is one of the computation-intensive blocks [34]. A implementation using an integer rate interpolation is achieved by replacing the full size IFFT with a smaller IFFT and a small interpolator, the interpolation-based SC-FDMA (I-SC-FDMA) presented in Fig. 1(b), can provide a significant complexity reduction when the resource allocation size is relatively small [35].

In [36], the authors demonstrate that this implementation allows a significant reduction in computational complexity when the size of the resource allocation is relatively smaller than the total number of subcarriers. ie, if this condition is met, I-SC-FDMA offers a reduction in complexity, otherwise C-SC-FDMA is preferred. It should be emphasized that the selection threshold is sufficiently high for a user in multi-user scenarios.

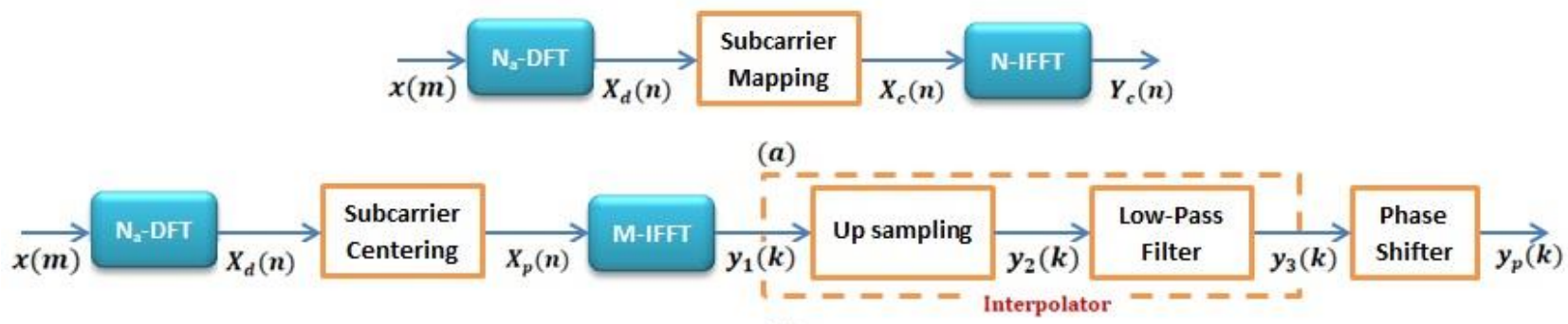

(b)

Fig. 1 - Simplified block diagrams for (a) conventional SC-FDMA transmitter; (b) Interpolation-based SCFDMA implementation [36]

\section{Modulations and Waveforms for 5G networks: CE-OFDM, FBMC, UFMC and F-OFDM}

The main disadvantage of OFDM is that the modulated waveform has high amplitude fluctuations that produce large peak-to-average power ratios (PAPR) [7]. The high PAPR increases the OFDM's sensitivity to non-linear distortion caused by the transmitter's power amplifier (PA) [37]. In the absence of sufficient power reduction, the system suffers from spectral broadening, intermodulation distortion and, therefore, performance degradation [38]. These problems can be reduced by applying different PAPR reduction methods [39], [40], but this has a negative effect on the PA efficiency [41]. For battery-powered mobile devices, this poses a real problem due to the limited energy resources. As a result, OFDM is considered inefficient in terms of power, which is undesirable especially for batterypowered wireless systems. Low energy consumption and good spectral efficiency are the most relevant expectations of $5 \mathrm{G}$.

In this section, we present candidate waveforms to meet the diversified and pronounced expectations of future cellular 5G networks, notably CE-OFDM to PAPR OdB for amplification without the need for linearization of the 
power amplifier. Moreover, FBMC, UFMC and F-OFDM as a modulation techniques that use different types of filters to improve the spectral efficiency of the system.

\subsection{Systems Models}

\subsubsection{FBMC System Model}

FBMC offers advantages that overcome the known limitations of OFDM in terms of reduced spectral efficiency and synchronization requirements. These advantages have led to consider FBMC as one of the modulation techniques of $5 \mathrm{G}$ communication systems. The Subcarrier Filtering approach used by FBMC to solve the ISI problem in OFDM is to add additional filtering at the transmitter and receiver levels side, next to IFFT/FFT blocks.

The blocks which constitute an FBMC system are presented in Fig. 2, where $T$ denotes the time interval of FBMC symbols and $N$ denotes the number of subcarriers [27]. For each subcarrier, the complex QAM symbols are first split into real parts and imaginary parts. The OQAM symbols are employed to avoid intrinsic interference in the conventional FBMC systems. Next to imaginary parts of a duration T/2, the joined OQAM signals come into the transmission filter, after that the signals will be modulated by the corresponding subcarrier frequency before being transmitted to the network. The receiver follows a reverse procedure to recover the signal sent by the transmitter.

A prototype filter that guarantees significant ISI suppression is obtained by introducing additional coefficients between the FFT coefficients in the frequency domain. In particular, the number of introduced coefficients between two consecutive DFT coefficients is called the "overlapping factor" of the filter $K$, which is also equal to the ratio between the filter impulse response duration and the multicarrier symbol period $T$, thereby determining the number of multicarrier symbols which overlap in the time domain. To guarantee a low ISI, the filter response must be impulse:

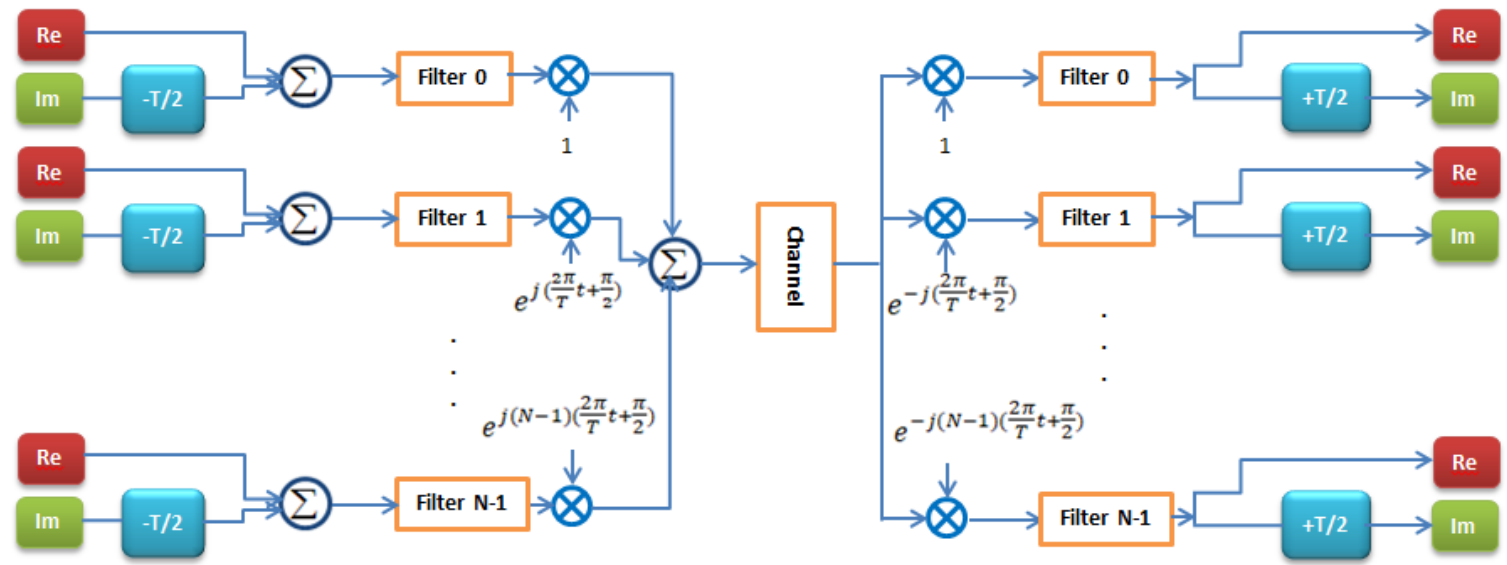

Fig. 3 - Block diagram for FBMC

$$
p(t)=1+2 \sum_{k=1}^{K-1} H_{k} \cos \left(2 \pi \frac{k t}{K T}\right)
$$

where the coefficients $H_{k}$ are given in Table. 1 , up to $K=4$.

Table 1 - Frequency-domain prototype filter coefficients

\begin{tabular}{ccccc}
\hline $\mathrm{K}$ & $\mathrm{H}_{0}$ & $\mathrm{H}_{1}$ & $\mathrm{H}_{2}$ & $\mathrm{H}_{3}$ \\
\hline 2 & 1 & $\sqrt{2} / 2$ & - & - \\
3 & 1 & 0.911438 & 0.411438 & - \\
4 & 1 & 0.971960 & $\sqrt{2} / 2$ & 0.235147
\end{tabular}

The complex input symbols are expressed as

$$
\begin{aligned}
X & =R_{m}^{N}+j I_{m}^{N}, \quad 0 \leq m \leq M-1 \text { and } 0 \leq n \leq N-1 \\
& =\left[\begin{array}{c}
R_{m}^{0}+j I_{m}^{0} \\
\cdot \\
\cdot \\
\cdot \\
R_{m}^{N-1}+j I_{m}^{N-1}
\end{array}\right], \quad 0 \leq m \leq M-1
\end{aligned}
$$


where $R_{m}^{n}$ and $I_{m}^{n}$ are the real and imaginary parts of the $m t h$ symbol on $n t h$ subcarrier, respectively.

The signal $s(t)$, consisting of $M$ symbols, sent over $N$ subcarriers can be expressed as

$$
s(t)=\sum_{m=0}^{M-1} \sum_{n=0}^{N-1} g_{m, n}(t) x_{m, n}
$$

where the basis pulse $g_{m, n}(t)=p(t-m T / 2) e^{j 2 \pi n F(t-m T)} e^{j \theta_{m, n}}$ is, essentially, a time and frequency shift version of the prototype filter $p(t) . x_{m, n}$ are real-valued symbols, which is related to $R_{m}^{n}$ and $I_{m}^{n}$ by the following equation.

$$
x_{2 m, n}=\left\{\begin{array}{ll}
R_{m}^{n} & n \text { is even } \\
I_{m}^{n} & n \text { is odd }
\end{array}, \quad x_{2 m+1, n}= \begin{cases}I_{m}^{n} & n \text { is even } \\
R_{m}^{n} & n \text { is odd }\end{cases}\right.
$$

In practical, the FBMC signal is generated by a sampling rate. Thus, the transmitted data of discrete time FBMC system can be written in a simple manner as

$$
s[i]=\sum_{m=0}^{M-1} \sum_{n=0}^{N-1} x_{m, n} g_{m, n}[i]
$$

where $g_{m, n}[i]$ denotes the shifted version used for the $n t h$ subcarrier. Considering an ideal channel with no noise, the demodulated symbol at the receiver for the $n^{\prime} t h$ sub-carrier and the $m^{\prime}$ th time slot can be written as

$$
\hat{x}_{m^{\prime}, n^{\prime}}=\sum_{i} \sum_{m=0}^{M-1} \sum_{n=0}^{N-1} x_{m, n} g_{m^{\prime}, n^{\prime}}^{*}[i] g_{m, n}[i]
$$

where $(.)^{*}$ denotes the complex conjugation.

The Power spectral density (PSD) of discrete random process of FBMC signal, is given in the following

$$
s(f)=|G(f)|^{2} A\left(\frac{K}{2} f\right)
$$

where $A\left(\frac{K}{2} f\right)$ is the PSD of the signal, $G(f)$ is the Fourier transform of the prototype filter $g[n]$, and $f$ is the normalized frequency with respect to the sampling frequency $f s$.

For the AWGN channel, the BER performance of FBMC system for 16-OQAM can be expressed as [42]

The SNR is defined as

$$
B E R_{F B M C}=\frac{1}{2}-\frac{3}{8 \sqrt{1+\frac{10}{S N R}}}-\frac{6}{8 \sqrt{9+\frac{10}{S N R}}}+\frac{5}{8 \sqrt{25+\frac{10}{S N R}}}
$$

$$
S N R=\frac{P_{x}^{O F D M}}{P_{n}}=\frac{P_{x}^{F B M C}}{\frac{1}{2} P_{n}}
$$

where $P_{x}=E\left\{\left|x_{m, n}\right|^{2}\right\}$ represents the average data symbol power. For the same bandwidth $F L$, setting $P_{x}^{F B M C}=\frac{1}{2} P_{x}^{\text {OFDM }}$ see (20), implies that the average transmit power $P_{s}=\frac{1}{K T} \int_{-\infty}^{+\infty} E\left\{|s(t)|^{2}\right\} d t$ is the same for OFDM, CP-OFDM and FBMC.

\subsubsection{UFMC System Model}

The waveform based on sub-band filtering is proposed to overcome the drawbacks of the subcarrier filtering used in FBMC. The sub-band-wise filtering is considered as a compromise between the whole band filtering of OFDM and subcarrier-wise filtering of FBMC. Hence, the filters are shorter compared to the FBMC, where the length of filters is much longer than the symbol duration. The total available bandwidth is partitioned into sub-bands and filtering is performed with a fixed frequency domain granularity.

The multi-carrier signal transmitted in the time domain for a user $\mathrm{k}$ is the superposition of the components filtered under the band, with the filter length $\mathrm{L}$ and the FFT length $N$. The signal demodulation in the receiver depends on a $2 N$ point FFT. A key feature of UFMC is the employment of that $2 N$-point FFT in the receiver, which can recover the data 
symbols without the need of CP. Given the length of the transmitted signal, $N$, and the length of the channel impulse response (CIR), $L$, the length of the received signal will be $N C L-1$. The data symbols can be recovered via the application of a $2 N$ point FFT followed by decimation with factor two after the filling of $N-L-1$ zeros at the end of the received signal. For more details see reference [27]. A block diagram of a conventional UFMC transmitter-receiver is shown in Fig. 3.

The transmitted signal is given by Equation (21)

$$
x_{k}=\sum_{i=1}^{B} x_{i k}=\sum_{i=1}^{B} F_{i k} V_{i k} S_{i k}
$$

where $S_{i k}$ is the transmitted signal of the $i t h$ sub-band and $B$ is the total number of sub-bands. $V_{i k}$ represents the IFFT to eplitz matrix for the $i t h$ sub-band. Size of $V_{i k}$ is $N \times n i . F_{i k}$ is Chebyshev filter applied to the ith sub-band. Size of this vector is $(\mathrm{N}+\mathrm{L}-1) \times \mathrm{N}$. Size of $S_{i k}$ is ni $\times 1$ and $k=0 \ldots . . B$.

UFMC can be even used with different subcarrier spacing or filter times for users in different sub-bands. E.g. user 1 uses a particular FFT size $N 1$ and filter length $L_{1}$, user 2 uses $N_{2}$ and $L_{2}$ and the UFMC symbol durations might be designed identically, thus $N_{1}+L_{1}-1=N_{2}+L_{2}-1$. This allows UFMC to be a highly adaptive modulation scheme, which can be easily tailored to many different aspects of communications such as delay/Doppler spread characteristics of the radio channel and user needs. A conventional UFMC receiver utilizes an FFT block that has twice the size of IFFT block at the transmitter. A Chebyshev window with parameterized side lobe attenuation is employed to filter the IFFT output per sub-band [13].

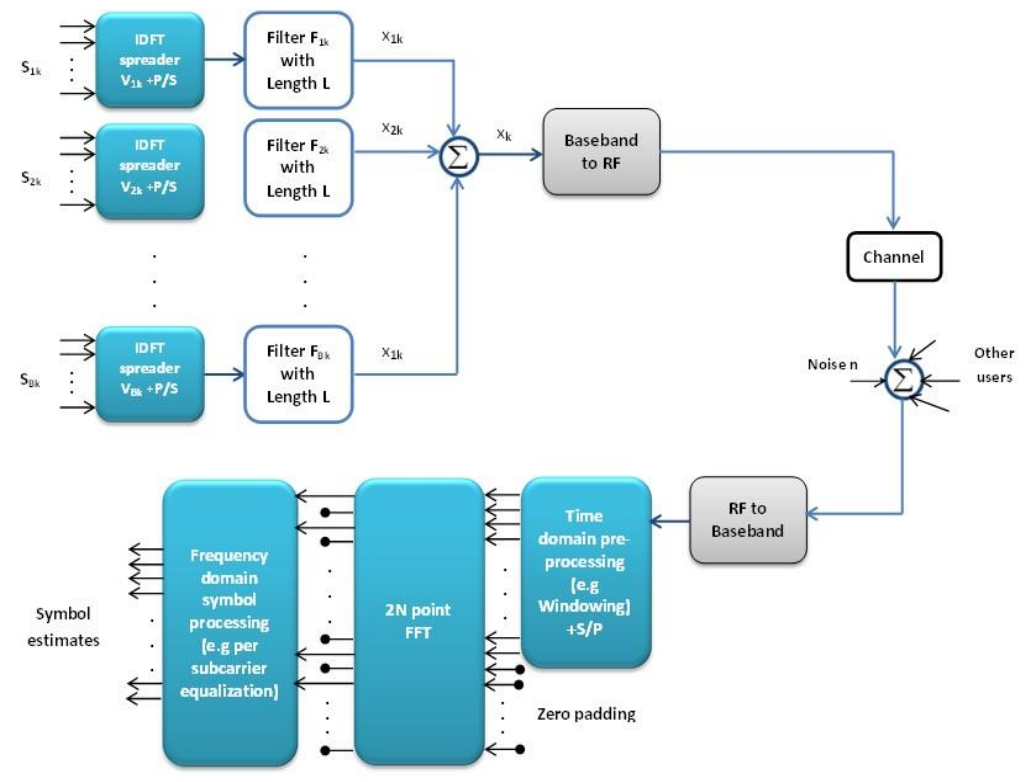

Fig. 3 - Basic concept of UFMC

For AWGN channel, the BER of OFDM using M-QAM in [43] is given as

$$
P_{\text {OFDM }}=\frac{2(\sqrt{M}-1)}{\sqrt{M} \log _{2} \sqrt{M}} Q\left(\sqrt{\frac{6 E_{b}}{N_{0}} \cdot \frac{\log _{2} \sqrt{M}}{M-1}}\right)
$$

where $M$ represents the modulation order, $E b / N 0$ is signal to noise ratio and $Q(x)$ is Q-function which is defined as

$$
Q(x)=\frac{1}{\sqrt{2 \pi}} \int_{x}^{+\infty} e^{-\frac{t^{2}}{2} d t}
$$

Unlike OFDM where filtering is applied to all of the symbol's sub-carriers. In UFMC, each subcarrier is filtered. On the receiver side, each sub-band must perform the reverse process of the filter. In this process, the noise variance on the $k$-th subcarrier of UFMC system in the $i$-th sub-band is

$$
\sigma_{k}^{2}=\frac{\sigma_{k}^{2}}{\left|r_{n}\right|^{2}}
$$


where $r_{k}$ indicates the equivalent filter response corresponding to the $k$-th subcarrier, and $\sigma_{n}$ represents Gaussian white noise variance. Therefore, the BER of M-QAM in the UFMC system under the AWGN channel can be expressed as

$$
P_{U F M C}=\frac{1}{K B} \sum_{i=1}^{B} \sum_{k=1}^{K} \frac{2(\sqrt{M}-1)}{\sqrt{M} \log _{2} \sqrt{M}} Q\left(\sqrt{\frac{6 E_{b}}{N_{0}}\left|r_{k}\right|^{2} \cdot \frac{\log _{2} \sqrt{M}}{M-1}}\right)
$$

where $K$ is the total subcarriers of each sub-band, and $B$ is the number of sub-bands. Although the UFMC is not an orthogonal waveform as a whole, the sub-bands and the subcarriers in the band are still orthogonal. Therefore, the overall BER of the system is equal to the average bit error rate of the respective sub-bands under the condition that each sub-band is independent of each other.

The BER of the system corresponding to the AWGN channel is the upper limit of the performance of the UFMC system. In fact, a communication system is often accompanied by fading channels. For UFMC, the main factor affecting the BER of system is the signal to noise ratio. After equalization compensation, the effect of channel on noise is the same for OFDM and UFMC. The only difference is that the filtering of the UFMC changes the power of the noise. Thus, the noise variance on the $k$-th subcarrier in the $i$-th sub-band for UFMC system can be written as

$$
\sigma_{k}^{2}=\frac{\sigma_{n}^{1}}{\left|\lambda_{k}\right|^{2}}
$$

where $\lambda_{k}$ is corresponded to the equivalent filter impulse response and the Rayleigh fading channel response in the $k$-th subcarrier, and $\sigma_{n}$ is the variance of Gaussian white noise. Bringing (23) to (22), the BER of UFMC can be rewritten as

$$
P_{U F M C}^{\prime}=\frac{1}{K B} \sum_{i=1}^{B} \sum_{k=1}^{K} \frac{2(\sqrt{M}-1)}{\sqrt{M} \log _{2} \sqrt{M}} E\left[Q\left(\sqrt{\frac{6 E_{b}}{N_{0}}\left|\lambda_{k}\right|^{2} \cdot \frac{\log _{2} \sqrt{M}}{M-1}}\right)\right]
$$

From the Eq. (38) the BER of UFMC is mainly determined by modulation order, signal to noise ratio, equivalent filter response, and fading channel response, which is different from OFDM. Simultaneously, the number of subcarriers $K$ and the number of sub-bands $B$ also impact on BER for UFMC. Under certain conditions, $\left|\lambda_{k}\right|^{2}=1$, OFDM and UFMC should have the same performance. But, in a practical implementation, we can't guarantee that this situation will be met. Under these circumstances, the performance of UFMC will not coincide with OFDM. This is due to the amplification of the noise caused by the filtering operation and channel equalization.

\subsubsection{F-OFDM System Model}

Filtered-OFDM (F-OFDM) is another sub-band-wise filtered multicarrier scheme, but the filtering granularity is more flexible than UFMC. According to the diagram in Fig. 4, F-OFDM is a waveform that uses full-band filtering, only one emission filter and another reception filter are used.

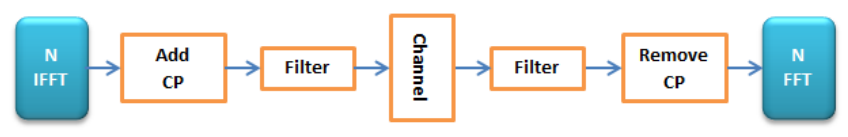

Fig. 4 - System structure of F-OFDM

The filtered frequency orthogonal frequency division multiplexing system (F-OFDM) is a promising waveform for $5 \mathrm{G}$ and beyond that allows multi-service and efficient spectrum slicing.

The F-OFDM shares all the advantages of well-localized frequency waveforms such as the low OOBE, allowing asynchronous transmission, requiring fewer guard tones and compatible with the MIMO transmission scheme. However, complexity remains the main drawback of F-OFDM compared to CP-OFDM.

\subsubsection{CE-OFDM System Model}

CE-OFDM is multi-carrier system which used to avoid the PAPR problem associated with OFDM system by way of signal transformation. This method consists of a transformation of the OFDM signal before the amplification part, at the receiver an inverse conversion is performed before the demodulation stage. By using this method we can reduce the PAPR to 0 dB. Fig. 5 gives the Block diagram of CE-OFDM system. 


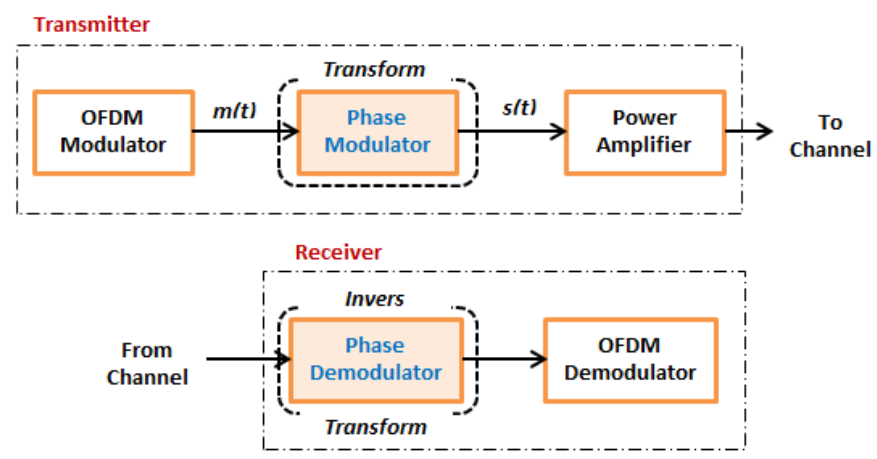

Fig. 5 - Basic concept of CE-OFDM

The baseband OFDM waveform

$$
m(t)=\sum_{i} \sum_{k=1}^{N} I_{i, k} q_{k}\left(t-i T_{B}\right)
$$

where $\left\{I_{i, k}\right\}$, are the data symbols and $\left\{q_{k}(t)\right\}$ are the orthogonal subcarriers. For CE-OFDM, before amplification $m(t)$ is passed through a phase modulator.

The baseband CE-OFDM signal is

$$
s(t)=A e^{j \phi(t)}
$$

where $A$ is the signal amplitude and $\phi(t)$ is the information bearing phase signal. The advantage of the CE waveform is that the instantaneous power is constant $|s(t)|^{2}=A^{2}$. Consequently, the PAPR is $0 \mathrm{~dB}$ and the required back off is 0 $\mathrm{dB}$.

The PA can therefore operate at the optimum (saturation) point, maximizing average transmit power (good for range) and maximizing PA efficiency (good for battery life).

The phase signal during ith block is written as

$$
\phi(t)=\theta_{i}+2 \pi h C_{N} \sum_{k=1}^{N} I_{i, k} q_{k}\left(t-i T_{B}\right), \quad i T_{B} \leq t<(i+1) T_{B}
$$

where $h$ is referred as the modulation index and $\theta_{i}$ is a memory term. The normalizing constant, $C_{N}$, is set to

$$
C_{N}=\sqrt{\frac{2}{N \sigma_{I}^{2}}}
$$

where $\sigma_{I}^{2}$ is the data symbol variance:

$$
\sigma_{I}^{2}=E\left\{\left|I_{i, k}\right|^{2}\right\}=\frac{M^{2}-1}{3}
$$

which is only a function of the modulation index. The signal energy is

$$
\varepsilon_{s}=\int_{i T_{B}}^{(i+1) T_{B}}|s(t)|^{2} d t=A^{2} T_{B}
$$

and the bit energy is

$$
\varepsilon_{b}=\frac{\varepsilon_{s}}{N \log _{2} M}=\frac{A^{2} T_{B}}{N \log _{2} M}
$$

CE-OFDM requires a real-valued OFDM message signal. Therefore, the data symbols in (28) are real-valued: 


$$
I_{i, k} \in\{ \pm 1, \pm 3, \ldots, \pm(M-1)\}
$$

This one-dimensional constellation is known as pulse-amplitude modulation (PAM). The subcarriers $\left\{q_{k}(t)\right\}$ must also be real-valued. Three possibilities are considered: half-wave cosines, half-wave sines, and full-wave cosines and sines.

In terms of implementation, (36) can be computed with a discrete cosine transform (DCT); (37) with a discrete sine transform (DST); and (38) by taking the real part of a discrete Fourier transform (DFT), or equivalently by taking a $2 \mathrm{~N}$-point DFT of a conjugate symmetric data vector as processed in [15].

For $k=1,2, \ldots, N$, half-wave cosines is,

$$
q_{k}(t)=\left\{\begin{array}{cc}
\cos \pi k t / T_{B} & 0 \leq t<T_{B} \\
0 & \text { othewise }
\end{array}\right.
$$

For $k=1,2, \ldots, N$, half-wave sines is,

$$
q_{k}(t)=\left\{\begin{array}{cc}
\sin \pi k t / T_{B} & 0 \leq t<T_{B} \\
0 & \text { othewise }
\end{array}\right.
$$

For $k=1,2, \ldots, N$ a full-wave cosines and sines is:

$$
q_{k}(t)=\left\{\begin{array}{cc}
\cos 2 \pi k t / T_{B} & 0 \leq t<T_{B} ; k \leq N / 2 \\
\sin 2 \pi(k-N / 2) / T_{B} & 0 \leq t<T_{B} ; k>N / 2 \\
0 & \text { othewise }
\end{array}\right.
$$

The smoothest phase results from CP-DCT (Continuous Phase-DCT)which, unlike DST and CP-DFT, has a first derivative equal to zero at the boundary times $t=i T_{B}$. Consequently, the CP-DCT is the most spectrally contained.

The power spectral density of the CE-OFDM signal can be easily estimated by Welch's method [44].

\subsection{Spectral Efficiency}

In order to answer the requirements of the applications that must be provided by $5 \mathrm{G}$ technology, spectral efficiency

\begin{tabular}{|c|c|}
\hline Spectral Efficiency & Parameters description \\
\hline \multicolumn{2}{|c|}{ OFDM } \\
\hline$S E_{\text {OFDM }}=\frac{R_{\text {OFDM }}}{W_{\text {OFDM }}}=\frac{N \log _{2} M}{N+N_{C P}}$ & $\begin{array}{l}R_{O F D M}: \text { Transmission rate } \\
W_{\text {OFDM }}: \text { Transmission bandwidth } \\
N_{\text {Number of subcarrier }} \\
N_{C P}: \text { Cyclic Prefix length } \\
M: \text { Modulation order } \\
\end{array}$ \\
\hline \multicolumn{2}{|c|}{ UFMC } \\
\hline$S E_{U F M C}=\frac{R_{U F M C}}{W_{U F M C}}=\frac{N \log _{2} M}{N+L-1}$ & $\begin{array}{l}R_{U F M C}: \text { Transmission rate } \\
W_{U F M C}: \text { Transmission bandwidth } \\
N: \text { Number of subcarrier } \\
L: \text { length of filter } \\
M: \text { Modulation order }\end{array}$ \\
\hline \multicolumn{2}{|c|}{ FBMC } \\
\hline$S E_{F B M C}=\frac{R_{F B M C}}{W_{F B M C}}=\frac{S \log _{2} M}{S+K-1 / 2}$ & $\begin{array}{l}R_{F B M C}: \text { Transmission rate } \\
W_{F B M C}: \text { Transmission bandwidth } \\
S: \text { Number of symbol } \\
K: \text { Overlapping factor } \\
M: \text { Modulation order }\end{array}$ \\
\hline \multicolumn{2}{|c|}{ CE-OFDM } \\
\hline \multirow[b]{2}{*}{$S E_{C E-O F D M}=\frac{R_{C E-O F D M}}{W_{C E-O F D M}}=\frac{\log _{2} M}{\max (2 \pi h, 1)}(42)$} & $R_{C E-O F D M}=\frac{N \log _{2} M}{T_{B}}(b / s)$ \\
\hline & $\begin{array}{l}T_{B}: \text { OFDM block period } \\
W_{F B M C}: \text { Transmission bandwidth } \\
h: \text { Modulation index } \\
M: \text { Modulation order }\end{array}$ \\
\hline
\end{tabular}
is a very important parameter. Good spectral efficiency increases the transmission capacity which has an effect on the data rate. Spectral efficiency, is can be defined as symbol transmission rate in a band of frequency.

Table 2 - Spectral efficiency of OFDM, UFMC, FBMC and CE-OFDM 
Through (39), (40), (41) and (42), it is obvious that with the reduction of cyclic prefix or the length of filter or the increasing of modulation order, the spectral efficiency of multicarrier modulation can be improved.

Note that, F-OFDM maintains the same CP-OFDM process and uses FIR filtering. Filtering does not reduce spectral efficiency. Therefore, the spectral efficiency of F-OFDM is also equal to that of CP-OFDM.

\section{Simulation Results and Comparison}

In this section, the simulation results and discussions regarding the comparison of power spectral density and BER performance in different candidate waveforms for 5G technology will be presented. The simulations are done by MATLAB. The simulation parameters for each system model are given in the Table. 3.

Table 3 - Systems parameters

\begin{tabular}{|c|c|c|}
\hline Parameter & Parameter description & Value \\
\hline \multicolumn{3}{|c|}{ OFDM parameters } \\
\hline $\bar{N}$ & Number of subcarrier & 128 \\
\hline$N_{C P}$ & Cyclic Prefix length & $N / 4$ \\
\hline numFFT & FFT size & 1024 \\
\hline \multirow{2}{*}{$S N R$} & Signal-to-noise ratio & $0-15$ \\
\hline & Modulation type & 16-QAM \\
\hline \multicolumn{3}{|c|}{ CE-OFDM parameters } \\
\hline $\bar{N}$ & Number of subcarrier & 128 \\
\hline$N_{C P}$ & Cyclic Prefix length & $N / 4$ \\
\hline$J$ & oversampling factor & 8 \\
\hline numFFT & FFT size & $N^{*} J$ \\
\hline $2 \pi h$ & $\mathrm{~h}:$ modulation index & $0.2-1.2-2$ \\
\hline \multirow[t]{2}{*}{$S N R$} & Signal-to-noise ratio & $0-15$ \\
\hline & Modulation type & 16-QAM \\
\hline \multicolumn{3}{|c|}{ FBMC parameters } \\
\hline$N$ & Number of subcarrier & 128 \\
\hline numFFT & FFT size & 1024 \\
\hline \multirow[t]{2}{*}{ SNR } & Signal-to-noise ratio & $0-15$ \\
\hline & Modulation type & 16-QAM \\
\hline \multirow[t]{2}{*}{$K$} & Overlapping Factor & 4 \\
\hline & Filter type & Prototype \\
\hline \multicolumn{3}{|c|}{ UFMC parameters } \\
\hline $\bar{N}$ & Number of subcarrier & 128 \\
\hline numFFT & FFT size & 1024 \\
\hline \multirow[t]{2}{*}{$S N R$} & Signal-to-noise ratio & $0-15$ \\
\hline & Modulation type & 16-QAM \\
\hline \multirow[t]{2}{*}{$B$} & Number of sub-band & 10 \\
\hline & Subcarrier per sub-band & 80 \\
\hline \multirow[t]{4}{*}{$L$} & Length of the sub-band filters & 33,50 \\
\hline & Filter side-lobe Attenuation & 40 \\
\hline & Filter type & Chebyshev \\
\hline & \multicolumn{2}{|l|}{ F-OFDM parameters } \\
\hline$N$ & Number of subcarrier & 128 \\
\hline$N_{C P}$ & Cyclic Prefix length & $N / 4$ \\
\hline \multirow[t]{3}{*}{$L$} & Filter length & 513 \\
\hline & Number of resource blocks & 64 \\
\hline & Bit per resource block & 8 \\
\hline
\end{tabular}

\subsection{CE-OFDM Performances}

In this section, the effect of the modulation order and modulation index $h$ on the BER performance of the CEOFDM system over the AWGN and Rayleigh channels are shown in Figures 6 and 7, respectively.

Figure 6 illustrates the effect of the modulation order $M(4,16,32,64)$ on the performance of the system, the simulation parameters of the proposed system are $\mathrm{N}=128,2 \pi \mathrm{h}=1$ and AWGN channel. From figure 6 it is clear that when $M=4$ the proposed system offers high performance compared to other modulation orders. However, the proposed system with $\mathrm{M}=64$ offers the highest throughput among the others but it offers also the highest BER. For example, to obtain a BER less than $10^{-3}$ we need a SNR more than $11 \mathrm{~dB}$ and $14 \mathrm{~dB}$ for $\mathrm{M}=4$ and $\mathrm{M}=16$ respectively. On other parameter that controls the performance of the proposed system is the modulation index. 


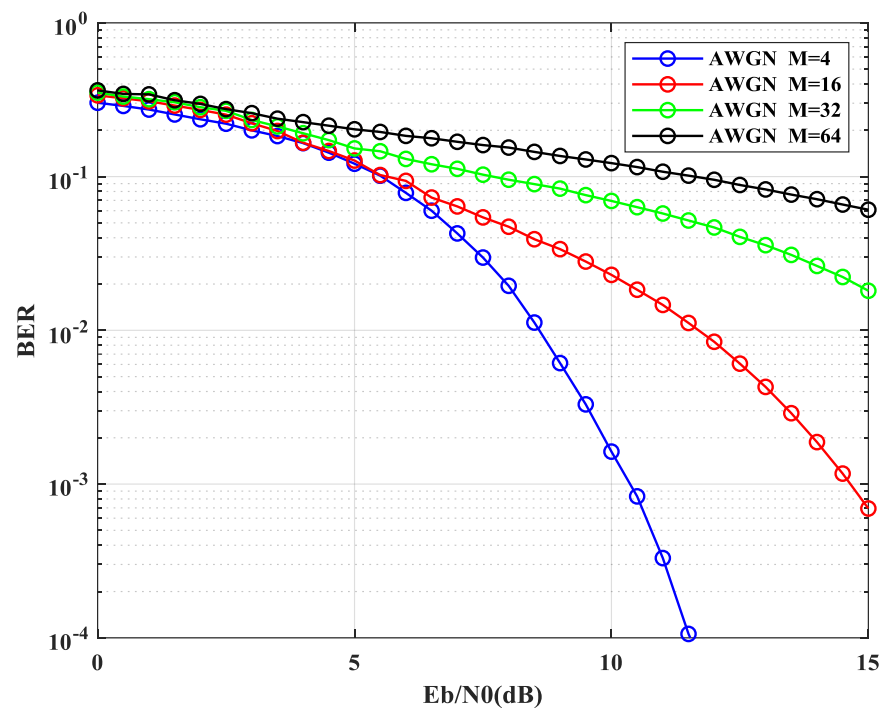

Fig. 6 - Modulation order effect on BER performance of CE-OFDM over AWGN channel

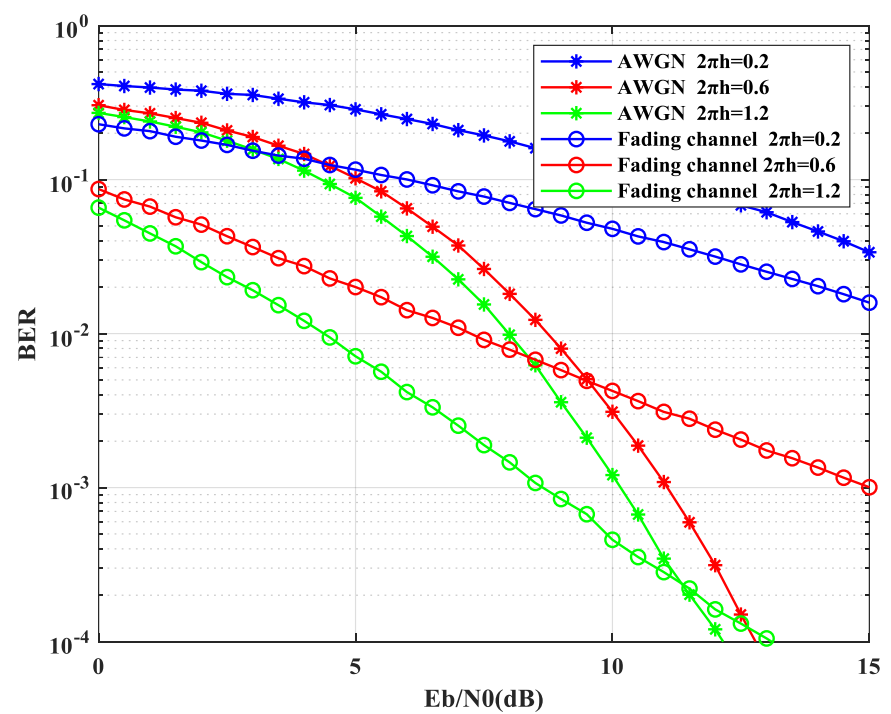

Fig. 7 - BER performance of CE-OFDM over AWGN and Fading channel

Figure 7 shows the BER as a function of the modulation index $\mathrm{h}$ (with $2 \pi \mathrm{h}=0.2,2 \pi \mathrm{h}=0.6$ and $2 \pi \mathrm{h}=1.2$ ) for $\mathrm{M}=$ $4, \mathrm{~N}=16$ and $\mathrm{J}=8$ (order of oversampling). Form this figure, it is clear that the CE-OFDM system has the best BER performance as $\mathrm{h}$ increases. Moreover, we notice that the performance of the proposed system over the AWGN channel is better compared to that obtained over the Rayleigh channel. The behavior of the system is better on the ideal channel AWGN except in the case of lower modulation index $(2 \pi \mathrm{h}=0.2)$.

\subsection{Power Spectral Density Comparison}

Power spectral density is a mathematical quantity that defines the spectral content of a signal. The PSD describes how a signal's power is distributed in frequency.

For the FBMC system, the order of the prototype filter can be chosen as follows: $2 * K$ - 1 , where $K$ is the overlap factor, which is the number of overlapping multi-carrier symbols in the time domain. The values of parameter $K$ are indicated in Table. 1.

The implementation of an FBMC scheme requires a frequency extension by a factor $K$. Indeed, one possible implementation is based on a frequency spreading plus a $K N$-point IFFT at the transmitter, and on a $K N$-point FFT followed by a dispreading at the receiver. However, increasing the FFT size by a factor $K$ poses significant complexity issues. In order to reduce the computation complexity, it is possible to use a polyphase FFT network that does not require any frequency spreading, but to the detriment of certain additional processing operations. 


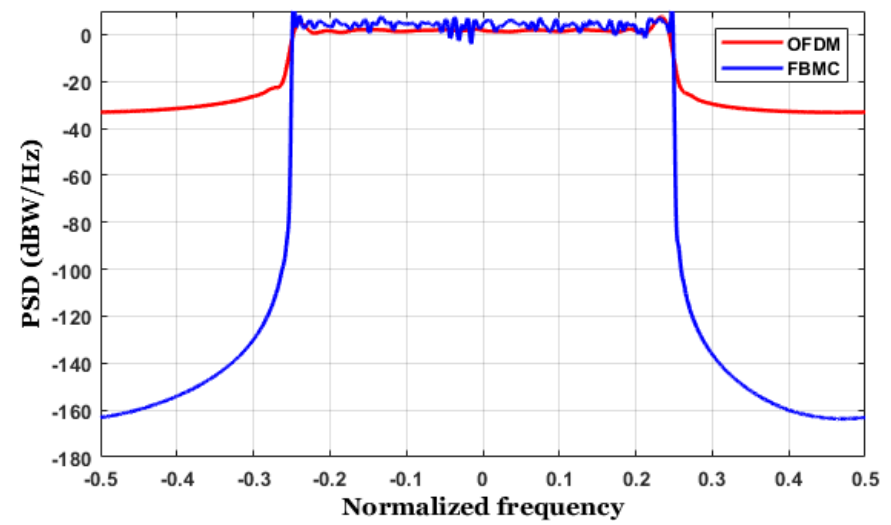

Fig. 8 - Power spectral density of FBMC versus OFDM

Fig. 8 shows the PSDs of the OFDM and FBMC-OQAM systems with the parameters shown in Table 2. The comparison of the power spectral density of the FBMC and OFDM systems shows the low out-of-band leakage of FBMC on OFDM. This is translated by: the lateral lobes of the FBMC-OQAM system are lower than those of the conventional OFDM systems, which improves spectral efficiency. The out-of-band radiation (OOB) is effectively reduced for FBMC than for OFDM.

In the next simulation, we highlight the advantages of the F-OFDM modulation scheme and compare it with the OFDM system, with cyclic prefix (CP-OFDM). We use a filter in the time domain to improve the out-of-band behavior while preserving the orthogonality of the symbols OFDM. For the implementation of F-OFDM, F-OFDM filtering must meet requirements such as 1)- A flat bandwidth on the sub-carriers of the sub-band 2)- A clear transition band to minimize guard-bands and 3)- Sufficient attenuation of the stopping band.

A rectangular frequency response filter, i.e. a sincere impulse response, meets these criteria. To create this causal link, the low-pass must be created using a window that effectively truncates the impulse response and provides smooth transitions to zero at both ends. Fig. 9 compares filtered OFDM modulation with the conventional OFDM system in terms of power spectral density.

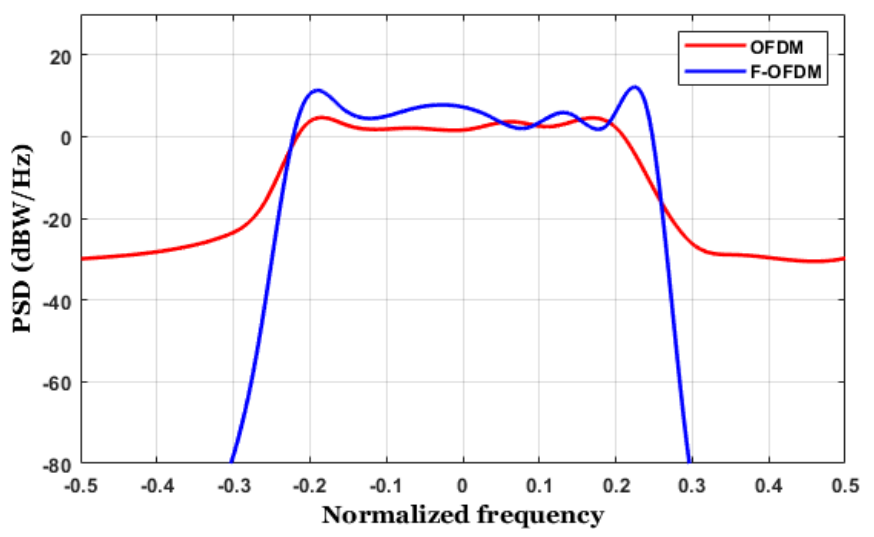

Fig. 9 - Power Spectral Density of F-OFDM versus OFDM

The simulation result presented in Fig. 9 shows that the F-OFDM has lower sidelobes than the OFDM system. It is clear that the out-of-band radiation (OOB) is effectively reduced for F-OFDM than for OFDM, which means that the spectral efficiency of F-OFDM is improved compared to that of OFDM.

Another technique that was studied in this work is the UFMC waveform. This technique is seen as a generalization of F-OFDM and FBMC modulations. The subcarrier grouping in UFMC allows one to reduce the filter length (when compared with FBMC). On the other hand, UFMC can still use QAM because it retains complex orthogonality compared to FBMC which works with MIMO schemes.

In UFMC the full band of subcarriers $N$ is divided into sub-bands. Each sub-band has a fixed number of subcarriers and not all sub-bands need to be employed for a given transmission. To filter the sub-bands, we can use different filters. However, in our case, the Chebyshev window with parameterized side lobe attenuation is used for all sub-bands. 

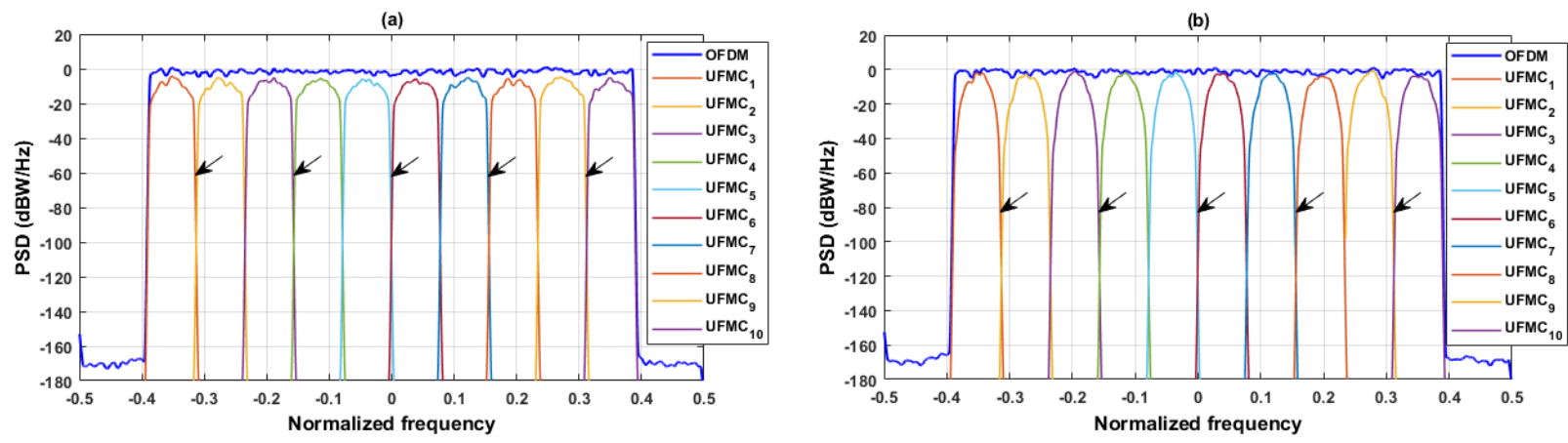

Fig. 10 - PSD of UFMC versus OFDM for different length of filter (a) $L=33$; (b) $L=50$

Using the parameters indicated in Table 2, the comparison of UFMC and OFDM systems of the same size IFFT with 4-QAM is presented in Fig. 10. This figure shows that the spectral density for the UFMC scheme has lateral lobes lower than those of the OFDM scheme. This allows greater use of the allocated spectrum, which leads to good spectral efficiency. Moreover, for each user in the UFMC system, the spectral roll-off is much more rapid. This rapid roll-off is an important advantage in sub-band multiplexing, as it means that there is much less interference between the different users of UFMC.

From Fig. 10(a) and Fig. 10(b), it can be concluded that the spectrum of the UFMC depends on the length of the filter used. A longer filter will result in more rapid spectral roll-off, at the cost of some loss in overall spectral efficiency and increase signal processing complexity. The UFMC waveform based on sub-band filtering can be used to serve low latency applications in a $5 \mathrm{G}$ network and to reduce the OOBE.

The last simulation is based on the CE-OFDM system. The latter is a complicated non-linear modulation and its power density spectrum can only be determined with estimates. The approach adopted to determine the power spectrum of conventional Continuous Phase Modulation (CPM) signals can be used to CE-OFDM. The modulation without memory $(\theta \mathrm{i}=0)$ leads to spectral lines at frequencies $f_{k}=k / T B, k=0, \pm 1, \pm 3, \ldots$ Using the memory eliminates these lines [45]. Different estimates can be used to determine the power spectral density of the CE-OFDM signal, such as Taylor's expansion and Abramson's spectrum. In our case, memory is used to guarantee the continuity of the spectrum.

The memory of CE-OFDM is defined by

$$
\theta_{i} \equiv \theta_{i-1}+K \sum_{k=1}^{N}\left[I_{i, k} A_{b}(k)-I_{i-1, k} A_{e}(k)\right]
$$
(28) is

The effective double-sided bandwidth, defined as the twice the highest frequency subcarrier, of $m(t)$ expressed in

$$
B_{m}=2 \times \frac{N}{2 T_{B}}=\frac{N}{T_{B}}
$$

The more appropriate and conservative bandwidth is $B_{s}=\max (2 \pi h, 1) B_{m}$
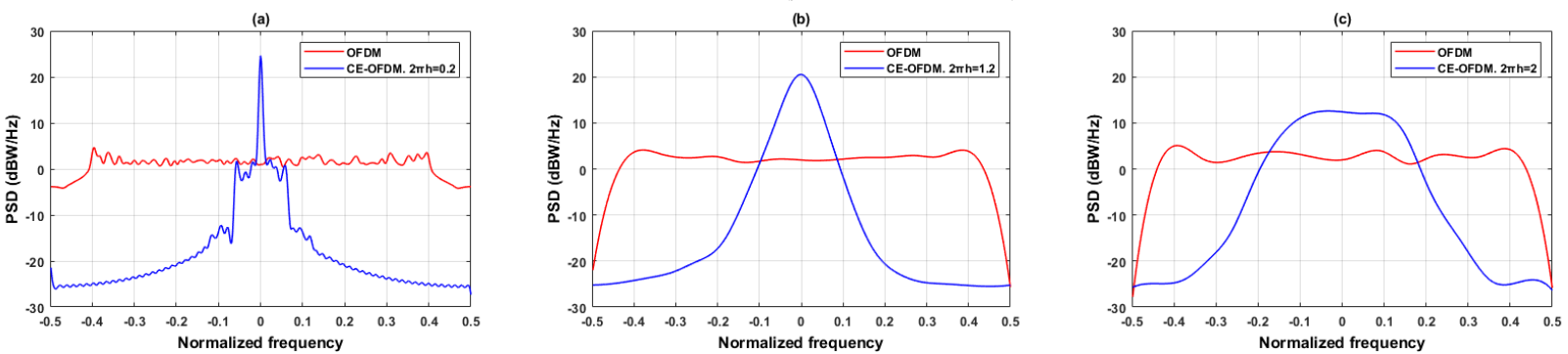

Fig. 11 - PSD of CE-OFDM versus OFDM for different values of $h$.

(a) $2 \pi h=0.2$; (b) $2 \pi h=1.2$; (c) $2 \pi h=2.0$

Fig. 11, shows that the modulation index controls the CE-OFDM spectral confinement. A smaller $\mathrm{h}$ can be used if a narrower spectrum is required. The trade-off is that $\mathrm{h}$ smaller results in poorer performance. Consequently and according to the needs, the designer of the system can exchange the performances against the spectral confinement and inversely. Due to CE-OFDM's phase-continuous design, exhibits faster side lobe roll-off than conventional OFDM. 


\subsection{BER Performance Comparison}

In this section we compare the performance of CE-OFDM, FBMC, UFMC, and F-OFDM waveforms in terms of BER. Figure 12 illustrates a comparison between the different waveforms cited above. From these results, we can note that the CE-OFDM wave form gives the best performance at high SNR. However, at low SNR FBMC is the efficient technique.

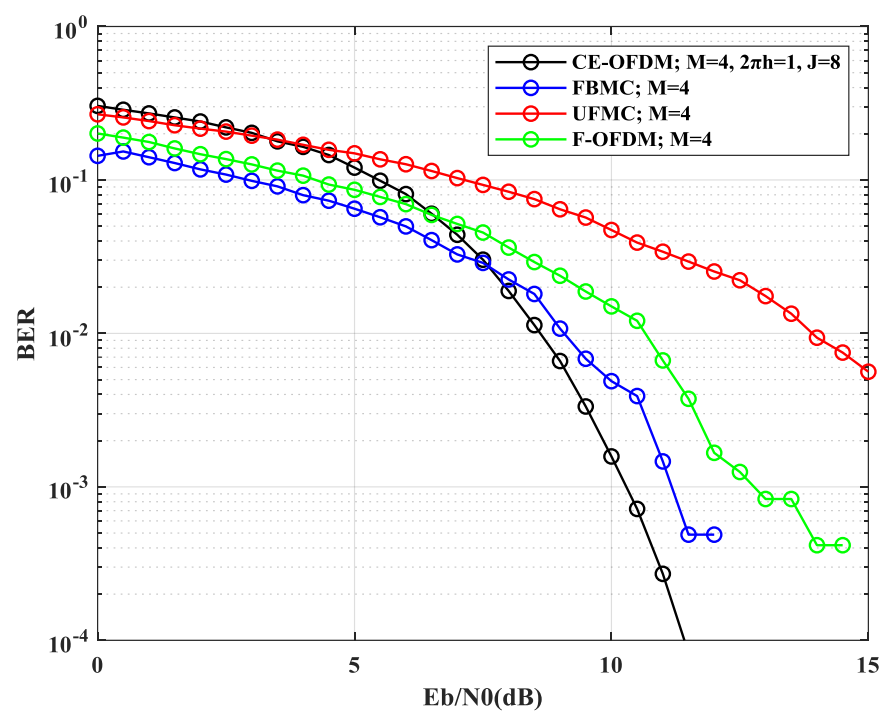

Fig. 12 - BER of CE-OFDM versus FBMC, UFMC and F-OFDM for M=4 over AWGN channel

However, as the desired BER is less than $10^{-3}$ the CE-OFDM waveform outperforms the FBMC one. For example, to obtain the desired BER we need a SNR of more than $10.5 \mathrm{~dB}$ and 11.5 for CE-OFDM and FBMC respectively. So, the CE-OFDM can be considered an appropriate technique for applications that need less BER. Furthermore, the two waveforms UFMC and F-OFDM are not suitable for applications that require less BER. To obtain a clear picture on the performance of the proposed wave forms let us see their performance over a fading channel. Figure 13 depicted the BER versus SNR of the wave forms cited before. It is clear form this figure that CE-OFDM is the desired technique over fading channel. For the others wave form, they can't offer a BER less that $10^{-3}$ even when BER more than $15 \mathrm{~dB}$.

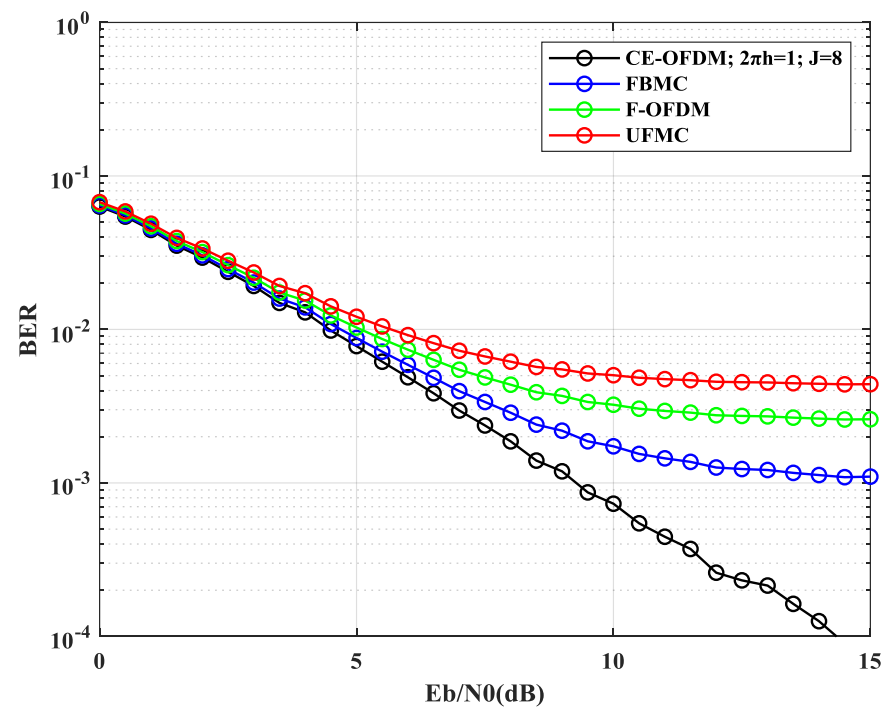

Fig. 13 - BER of CE-OFDM versus FBMC, UFMC and F-OFDM for M=4 over fading channel

\section{Conclusion}

$5 \mathrm{G}$ systems must meet the requirements of applications that require higher data rates, lower latency, and better spectrum exploitation. New waveforms are therefore envisaged for $5 \mathrm{G}$ communication systems to overcome some of these factors. In this paper, an overview of techniques that can be used for 5G applications such as CE-OFDM, FBMC, UFMC, and F-OFDM is presented. The grouping of subcarriers in UFMC can be considered as an intermediate case 
between the FBMC where the subcarriers are filtered individually and the F-OFDM in which the band is fully filtered. The simulation results show that the proposed techniques can be used in $5 \mathrm{G}$ applications that require high spectral efficiency and better power efficiency. Moreover, CE-OFDM it's a technique with a constant envelope that has the advantage to eliminate the PAPR associated with other waveforms, i.e. it is a less power consumption technique, which is desirable for battery-powered wireless systems. It has been demonstrated by simulation that the CE-OFDM outperforms the other technics in terms of performance. For this reason, CE-OFDM can be the best choice for $5 \mathrm{G}$ applications, especially for battery-powered devices.

\section{References}

[1] T. S. Rappaport et al. (2013). Millimeter wave mobile communications for 5G cellular: It will work!. IEEE access, vol. 1, pp. 335-349

[2] A. Ghosh. (2016). The 5G mmWave radio revolution. Microw. J, vol. 59, no. 9, pp. 22-36

[3] J. G. Andrews et al. (2014). What will 5G be?. IEEE J. Sel. areas Commun., vol. 32, no. 6, pp. 1065-1082

[4] Z. Pi and F. Khan. (2011). An introduction to millimeter-wave mobile broadband systems. IEEE Commun. Mag., vol. 49, no. 6, pp. 101-107

[5] M. Morelli. (2004). Timing and frequency synchronization for the uplink of an OFDMA system. IEEE Trans. Commun., vol. 52, no. 2, pp. 296-306

[6] R. Irmer et al. (2011). Coordinated multipoint: Concepts, performance, and field trial results. IEEE Commun. Mag., vol. 49, no. 2, pp. 102-111

[7] H. Ochiai and H. Imai. (2001). On the distribution of the peak-to-average power ratio in OFDM signals. IEEE Trans. Commun., vol. 49, no. 2, pp. 282-289

[8] G. Wunder et al. (2014). 5GNOW: non-orthogonal, asynchronous waveforms for future mobile applications. IEEE Commun. Mag., vol. 52, no. 2, pp. 97-105

[9] L. Zhang, P. Xiao, A. Zafar, A. ul Quddus, and R. Tafazolli. (2016). FBMC system: An insight into doubly dispersive channel impact. IEEE Trans. Veh. Technol., vol. 66, no. 5, pp. 3942-3956

[10] D. Chen, D. Qu, T. Jiang, and Y. He. (2012). Prototype filter optimization to minimize stopband energy with NPR constraint for filter bank multicarrier modulation systems. IEEE Trans. Signal Process., vol. 61, no. 1, pp. 159169

[11] V. Vakilian, T. Wild, F. Schaich, S. ten Brink, and J.-F. Frigon. (2013). Universal-filtered multi-carrier technique for wireless systems beyond LTE0 in 2013 IEEE Globecom Workshops (GC Wkshps), pp. 223-228

[12] X. Wang, T. Wild, and F. Schaich. (2015). Filter optimization for carrier-frequency-and timing-offset in universal filtered multi-carrier systems. in 2015 IEEE 81st Vehicular Technology Conference (VTC Spring), pp. 1-6

[13] X. Zhang, M. Jia, L. Chen, J. Ma, and J. Qiu. (2015). Filtered-OFDM-enabler for flexible waveform in the 5th generation cellular networks. in 2015 IEEE Global Communications Conference (GLOBECOM), pp. 1-6

[14] J. Abdoli, M. Jia, and J. Ma. (2015). Filtered OFDM: A new waveform for future wireless systems. in 2015 IEEE 16th International Workshop on Signal Processing Advances in Wireless Communications (SPAWC), pp. 66-70

[15] S. C. Thompson, A. U. Ahmed, J. G. Proakis, J. R. Zeidler, and M. J. Geile. (2008). Constant envelope OFDM. IEEE Trans. Commun., vol. 56, no. 8, pp. 1300-1312

[16] C.-D. Chung. (2010). Spectral precoding for constant-envelope OFDM. IEEE Trans. Commun., vol. 58, no. 2, pp. $555-567$

[17] A. U. Ahmed and J. R. Zeidler. (2015). Novel low-complexity receivers for constant envelope OFDM. IEEE Trans. Signal Process., vol. 63, no. 17, pp. 4572-4582

[18] Y. Li and D. Ding. (2018). Spectrum efficiency improvement for quasi-constant envelope OFDM. IEEE Photonics Technol. Lett., vol. 30, no. 15, pp. 1392-1395

[19] J. Belkadid, A. Benbassou, and M. El Ghzaoui. (2013). PAPR reduction in CE-OFDM system for numerical transmission via PLC channel. Int. J. Commun. Antenna Propag., vol. 3, no. 5, pp. 267-272

[20] T. F. Rahman, C. Sacchi, S. Morosi, A. Mazzinghi, and N. Bartolomei. (2018). Constant-envelope multicarrier waveforms for millimeter wave 5G applications. IEEE Trans. Veh. Technol., vol. 67, no. 10, pp. 9406-9420

[21] J. Mestoui, M. El Ghzaoui, M. Fattah, A. Hmamou, and J. Foshi. (2019). Performance analysis of CE-OFDMCPM Modulation using MIMO system over wireless channels. J. Ambient Intell. Humaniz. Comput., pp. 1-9

[22] M. El Ghzaoui, A. Hmamou, J. Foshi, and J. Mestoui. (2020). Compensation of Non-linear Distortion Effects in MIMO-OFDM Systems Using Constant Envelope OFDM for 5G Applications. J. Circuits, Syst. Comput., p. 2050257

[23] M. Van Eeckhaute, A. Bourdoux, P. De Doncker, and F. Horlin. (2017). Performance of emerging multi-carrier waveforms for $5 \mathrm{G}$ asynchronous communications. EURASIP J. Wirel. Commun. Netw., vol. 2017, no. 1, p. 29

[24] S. Wei, H. Li, W. Zhang, and W. Cheng. (2019). A comprehensive performance evaluation of universal filtered multi-carrier technique. IEEE Access, vol. 7, pp. 81429-81440

[25] B. Farhang-Boroujeny and H. Moradi. (2016). OFDM inspired waveforms for 5G. IEEE Commun. Surv. I\& Tutorials, vol. 18 , no. 4, pp. 2474-2492 
[26] J. Pérez Santacruz, S. Rommel, U. Johannsen, A. Jurado-Navas, and I. Tafur Monroy. (2020). Candidate waveforms for ARoF in beyond 5G. Appl. Sci., vol. 10, no. 11, p. 3891.

[27] Y. Liu et al. (2017). Waveform design for 5G networks: Analysis and comparison. IEEE Access, vol. 5, pp. 19282-19292

[28] R. Kaur, N. Kaur, and L. Kansal. (2018). Comprehensive study of future waveforms for 5G. Int. J. Pure Appl. Math., vol. 118, no. 20, pp. 4663-4672

[29] O. E. Ijiga, O. O. Ogundile, A. D. Familua, and D. J. J. Versfeld. (2019). Review of channel estimation for candidate waveforms of next generation networks. Electronics, vol. 8, no. 9, p. 956

[30] P. Goyal and A. K. Sahoo. (2019). A roadmap towards connected living: 5G mobile technology. Int. J. Innov. Technol. Explor. Eng, vol. 9, no. 1, pp. 1670-1685

[31] A. Ghosh, J. Zhang, J. G. Andrews, and R. Muhamed. (2010). Fundamentals of LTE. Pearson Education

[32] H. G. Myung, J. Lim, and D. J. Goodman. (2006). Single carrier FDMA for uplink wireless transmission. IEEE Veh. Technol. Mag., vol. 1, no. 3, pp. 30-38

[33] A. Ibing and V. Jungnickel. (2007). On hardware implementation of multiuser multiplexing for SC-FDMA. in 2007 IEEE 66th Vehicular Technology Conference, pp. 2056-2059

[34] Y.-W. Lin and C.-Y. Lee. (2007). Design of an FFT/IFFT processor for MIMO OFDM systems. IEEE Trans. Circuits Syst. I Regul. Pap., vol. 54, no. 4, pp. 807-815

[35] R. E. Crochiere and L. R. Rabiner. (1981). Interpolation and decimation of digital signals-A tutorial review. Proc. IEEE, vol. 69, no. 3, pp. 300-331

[36] H. Nam. (2010). Interpolation-based SC-FDMA transmitter with localized resource allocation. IEEE Commun. Lett., vol. 14, no. 10, pp. 948-950

[37] P. Banelli. (2003). Theoretical analysis and performance of OFDM signals in nonlinear fading channels. IEEE Trans. Wirel. Commun., vol. 2, no. 2, pp. 284-293

[38] P. Banelli, G. Baruffa, and S. Cacopardi. (2001). Effects of HPA nonlinearity on frequency multiplexed OFDM signals. IEEE Trans. Broadcast., vol. 47, no. 2, pp. 123-136

[39] K. Ramli et al. (2018). An enhanced partial transmit sequence based on combining Hadamard matrix and partitioning schemes in OFDM systems. Int. J. Integr. Eng., vol. 10, no. 3

[40] K. N. Ramli, M. A. Taher, N. S. M. Shah, L. H. Audah, M. S. Ahmed, and others. (2018). A New Subblock Segmentation Scheme in Partial Transmit Sequence for Reducing PAPR Value in OFDM Systems. Int. J. Integr. Eng., vol. 10, no. 8

[41] F. H. Raab et al. (2002). Power amplifiers and transmitters for RF and microwave. IEEE Trans. Microw. Theory Tech., vol. 50, no. 3, pp. 814-826

[42] R. Nissel and M. Rupp. (2017). OFDM and FBMC-OQAM in doubly-selective channels: Calculating the bit error probability. IEEE Commun. Lett., vol. 21, no. 6, pp. 1297-1300

[43] M. Agrawal and Y. Raut. (2011). BER analysis of MIMO OFDM system for AWGN $\backslash \&$ Rayleigh fading channel. Int. J. Comput. Appl., vol. 34, no. 9, pp. 33-37

[44] H. Dai and H. V. Poor. (2003). Advanced signal processing for power line communications. IEEE Commun. Mag., vol. 41, no. 5, pp. 100-107

[45] S. C. Thompson and others. (2005). Constant envelope OFDM phase modulation. Citeseer 